\title{
Análise preliminar dos dados magnéticos do Saco de Mamanguá e da Enseada de Paraty-Mirim, Rio de Janeiro
}

Janaína Anjos Melo, Yara Regina Marangoni e Luigi Jovane

Copyright 2019, SBGf - Sociedade Brasileira de Geofísica.

This paper was prepared for presentation at 16th International Congress of the Brazilian Geophysical Society, held in Rio de Janeiro, Brazil, 19-22 August 2019.

Contents of this paper were reviewed by the Technical Committee of the 16th International Congress of the Brazilian Geophysical Society. Ideas and concepts of the text are authors' responsibility and do not necessarily represent any position of the SBGf its officers or members. Electronic reproduction or storage of any part of this paper for commercial purposes without the written consent of the Brazilian Geophysical Society is prohibited.

\begin{abstract}
In the present work we investigate the magnetic anomalies located in the adjacent coves of the Paraty-Mirim and the Saco de Mamanguá, Rio de Janeiro, through marine magnetic and aeromagnetic data. In this preliminary study we filtered the data using upward continuation of 1500 meters and power spectrum analysis to characterize these anomalous. In addition, we obtain 3D magnetic models through the Geosoft VOXI Earth Modeling Cloud Inversion solution using the Magnetization Vector Inversion (MVI) technique. Through the interpretation of inversions we noticed anomalies with patterns of lineaments that extend beyond the study area. The presence of zones with distinct magnetic characteristics in a region of low magnetic latitude showed to be significant and encouraged hypotheses about the origin of these bodies.
\end{abstract}

\section{Introduction}

Segundo o trabalho de Maly (2017), foram detectadas feições geoelétricas indicativas de presença de gás em sedimentos rasos na Enseada de Paraty-Mirim (EPM) e no Saco de Mamanguá (SM). O estudo de estruturas anômalas em subsuperfície nas duas enseadas, EPM e o SM, utilizando a magnetometria, é o foco deste trabalho.

A análise de anomalias magnéticas com polaridade inversa tipicamente associadas a uma magnetização remanente, em que a direção de magnetização da fonte é paralela ao campo indutor, ainda é um desafio no contexto magnético.

Este trabalho tem como objetivo estudar a aplicabilidade da ferramenta VOXI, utilizando principalmente a técnica de Inversão do Vetor de Magnetização (MVI) em uma área de baixa latitude magnética. A inversão magnética consiste em calcular os parâmetros físicos a partir dos dados observados (Barbosa \& Pereira, 2013). Como há uma diversidade de modelos gerados, escolhe-se o que melhor representa e se correlaciona com a geologia da área investigada.

\section{Contexto Geológico}

A EPM caracteriza-se por se morfologicamente dendrítica e possui uma feição estreita e alongada de $11 \mathrm{~km}$ de comprimento. O SM apresenta uma feição de $2 \mathrm{~km}$ de largura aproximadamente. Estas enseadas são adjacentes e estão situadas ao extremo sudoeste da Baía de llha Grande, no estado do Rio de Janeiro. Compreendem a extensa feição geomorfológica Serra do Mar (fig. 1), (Maly, 2017).

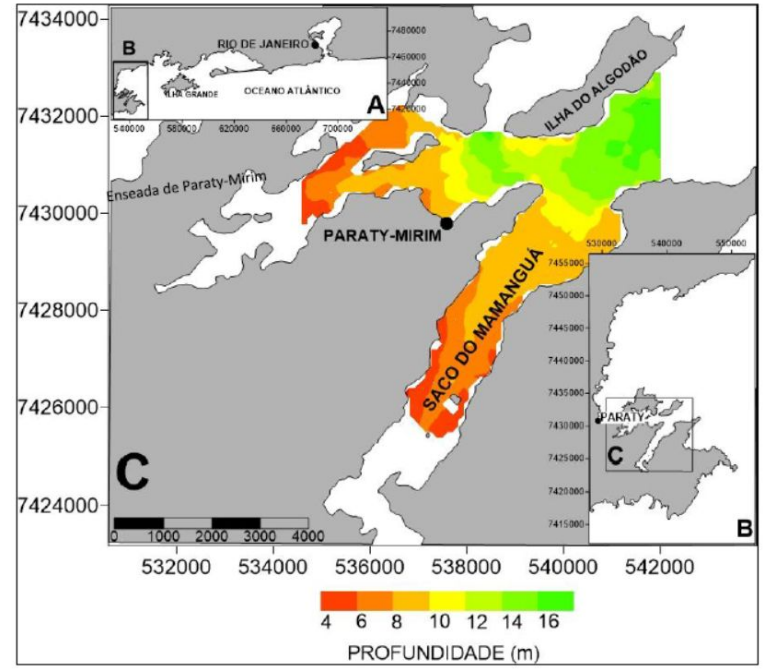

Figura 1: Localização da área de estudo - projeção UTM, datum WGS84, zona 23 S (Maly, 2017).

Os terrenos emersos que circundam as enseadas são formados por granitos e migmatitos pré-cambrianos que estão inseridos nas unidades estratigráficas: Granito Parati, Suíte Getulândia, Rio Negro, Granito Parati-Mirim e Suíte Serra das Araras, como apresentado na Fig. 2. Segundo a literatura (Telford et al. 1990, Blakely, 1995), espera-se que esses terrenos ígneos e metamórficos apresentem maiores valores de susceptibilidade magnética. 


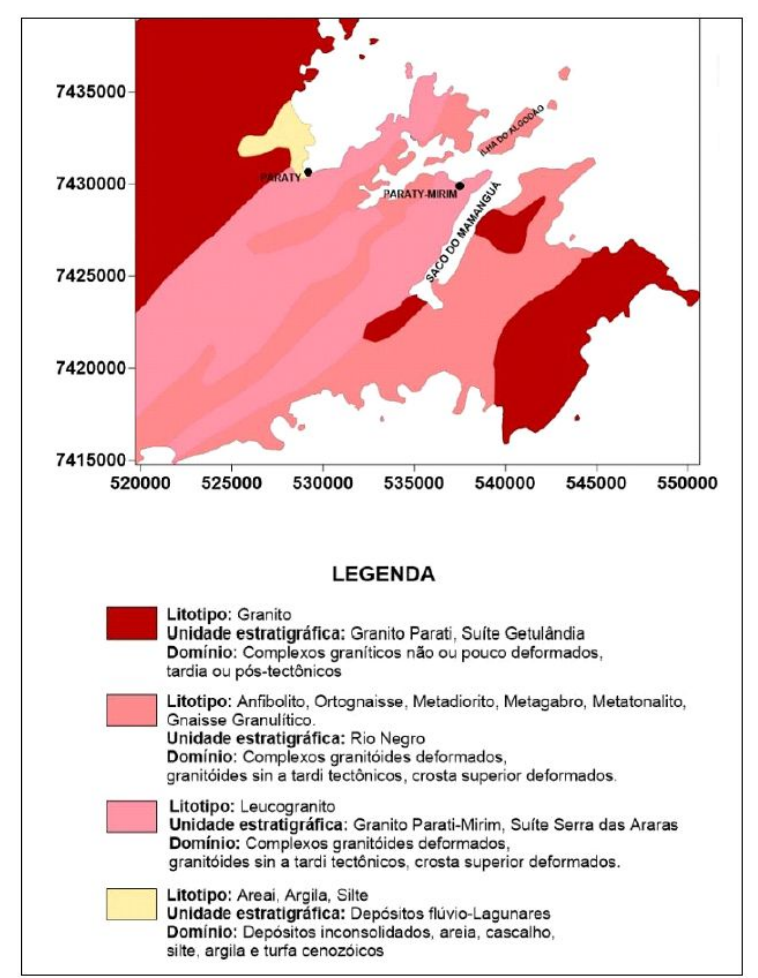

Figura 2: Mapa geológico dos terrenos emersos adjacentes às enseadas EPM e SM ( Maly, 2017).

De acordo com o Almeida \& Carneiro (1998), a diversidade de litotipos exibidos advém de uma história geológica compreendida entre o Pré-Cambriano e o Eopaleozóico (fig. 2). Além disso, estes autores afirmam que as datações mais antigas do embasamento costeiro abrangem o Arqueano até o Proterozóico Inferior.

Segundo Olpe (2016), a área de interesse exibe um regime de circulação limitada em uma região de micro maré com periodicidade semidiurna. O fundo é caracterizado por um relevo suave e abriga sedimentos finos, reproduzindo um ambiente em que os processos hidrodinâmicos operantes são de baixa energia e refletem um local de depocentro (Bernardes, 1996).

A disposição estratigráfica da região de estudo foi influenciada pelas variações do nível do mar ao longo do Quaternário, cujos indícios se situam, possivelmente, no arquivo sedimentar da área da Baía de Ilha Grande. Mahiques (1987) os dividiu em 5 eventos primordiais, que encadearam a paisagem conhecida atualmente.

\section{Method}

Este estudo utiliza dados magnéticos marinhos coletados pelo barco do Instituto Oceanográfico, Universidade de São Paulo, nos dias 6 e 7 de outubro de 2017 (fig. 3). O magnetômetro base estava situado em $23^{\circ} 15^{\prime} 47,31^{\prime \prime} \mathrm{S} 44^{\circ} 38^{\prime} 9,78^{\prime \prime} \mathrm{W}$, próximo ao Mamanguá Beach Hostel. O barco locomoveu-se a uma velocidade média de 8,9 nós. Um catamarã serviu como suporte para o SeaSpy, magnetômetro Overhauser de 0,001 nT de resolução e 0,1 nT de acurácia. A distância entre o sensor magnético (peixe) e o barco foi de 40 metros. O magnetômetro base fez uma medida a cada $30 \mathrm{~s}$, enquanto que o peixe fez uma medida a cada $1 \mathrm{~Hz}$. A altura do levantamento em relação ao nível do mar foi de 0 metros (fig. 4). Como mostrado na Fig. 4, a disposição das linhas do levantamento não é regular, o espaçamento entre elas variou entre 300 e 400 metros, salvo em algumas regiões em que elas estão mais próximas. A inclinação e declinação magnética eram de $-39,2^{\circ}$ e de $-22,2^{\circ}$, respectivamente.

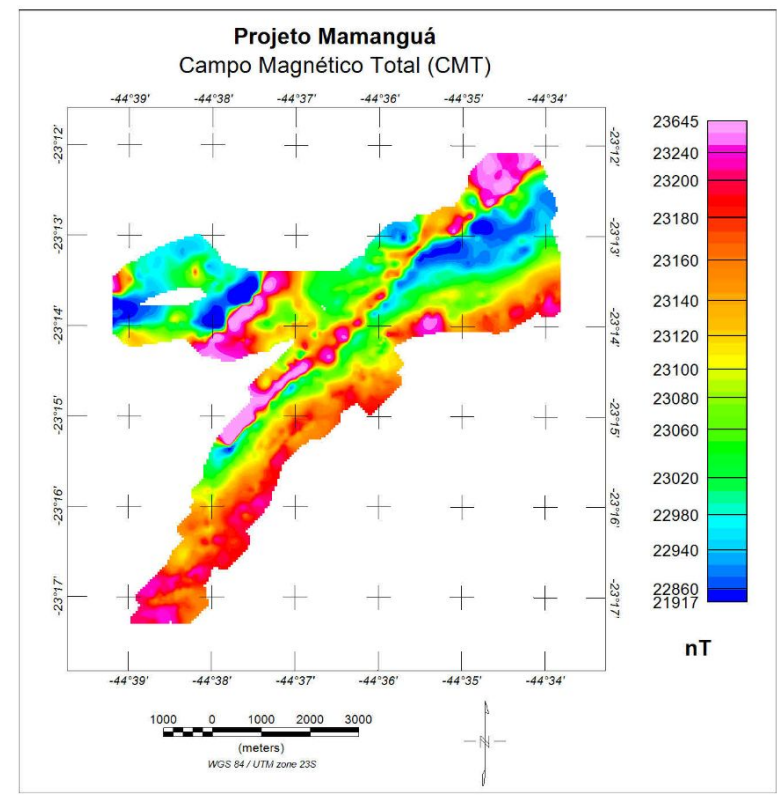

Figura 3: Mapa do campo magnético total (CMT) .

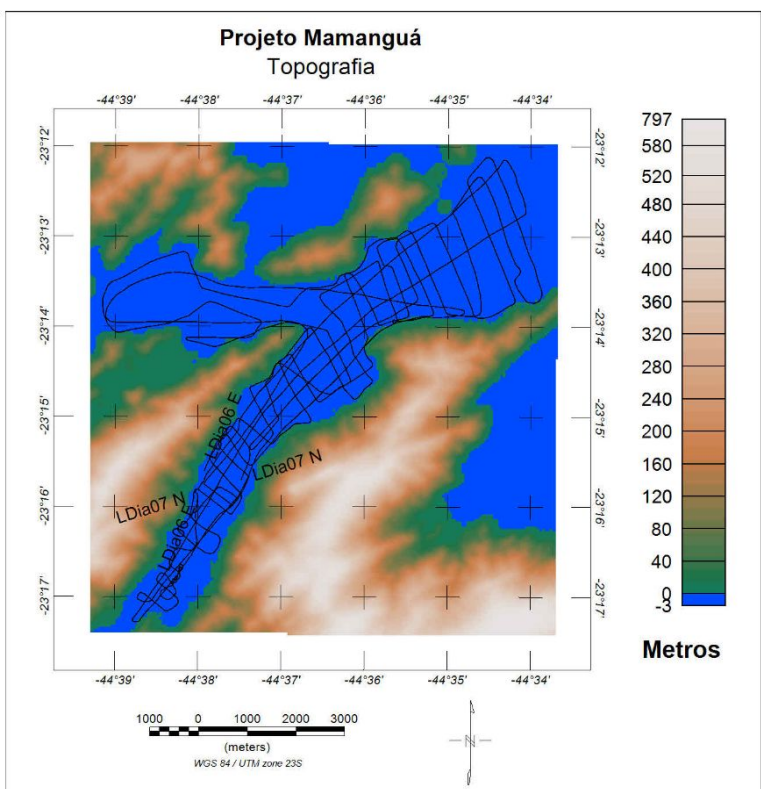

Figura 4: Representação da topografia da área de estudo com as linhas de aquisição.

Também são utilizados dados aeromagnetométricos obtidos pela CODEMIG em parceria com a CPRM em 2013. O levantamento aerogeofísico recobre uma área de $47.321 \mathrm{~km}^{2}$. As linhas de voo N-S e de controle E-W são espaçadas de $0,5 \mathrm{~km}$ e $10,0 \mathrm{~km}$, respectivamente. A altura de voo de $100 \mathrm{~m}$ sobre o terreno com 
tolerância de $+15 \mathrm{~m}$ por no máximo $2 \mathrm{~km}$ (CPRM/CODEMIG, 2013).

\section{Princípio Físico do MVI}

Os softwares usualmente empregados aplicam a inversão de susceptibilidade que se baseia na ideia dos domínios magnéticos se alinharem paralelamente ao campo geomagnético, entretanto, isso nem sempre ocorre (Barbosa \& Pereira, 2013).

O desenvolvimento do método MVI foi fundamental, pois permitiu a representação das propriedades magnéticas das rochas sem requerer um conhecimento prévio sobre a fonte e a direção da magnetização. Esta técnica considera não só a magnetização induzida como a remanência, a desmagnetização e a anisotropia dos materiais magnéticos a fim de calcular a amplitude e a direção dos domínios magnéticos (Barbosa \& Pereira, 2013).

O MVI fundamenta-se na combinação de duas componentes escalares do vetor de magnetização conhecido como Amplitude component - geradas na inversão, denominadas de Projected (paralela ao campo da Terra) e Perpendicular (perpendicular ao campo da Terra) components que auxiliam a analisar a importância do campo indutor e determinam como prosseguir na iteração do modelo (fig. 4) (Smith, 2018). Consequentemente, obtém-se um modelo 3D que representa de forma mais realista a complexa geologia em subsuperfície.

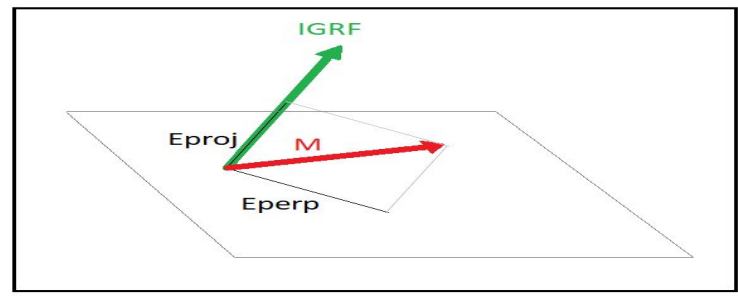

Figura 4: $O$ vetor de magnetização $(\mathrm{M})$, conhecido como Amplitude component, pode ser representado por duas componentes escalares: Projected component (Eproj) e Perpendicular component (Eperp).

\section{Results}

Na Fig. 5 estão apresentados os perfis da variação diurna do campo magnético monitorada pelo magnetômetro base do levantamento marinho. Nota-se que no dia $6 / 10$, comparado ao dia $7 / 10$, a variação diurna foi menos suave e pouco regular, logo, a atividade solar foi mais intensa neste dia. Foi realizada a correção diurna nos dados.
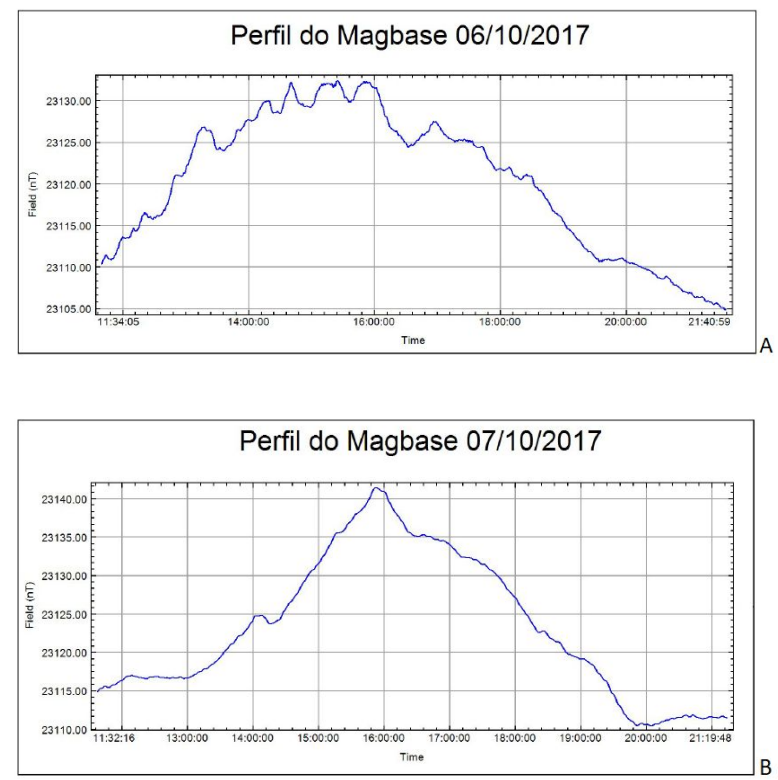

Figura 5: Perfis da variação diurna para os dias 6 e 7 de outubro.

A aplicação do filtro continuação para cima de $1500 \mathrm{~m}$ no campo magnético corrigido do IGRF permite observar as tendências regionais. Sendo assim, atenuou-se as anomalias de alta frequência e foram realçadas as anomalias de maior comprimento de onda, ou seja, mais profundas, como mostrado na Fig. 6.

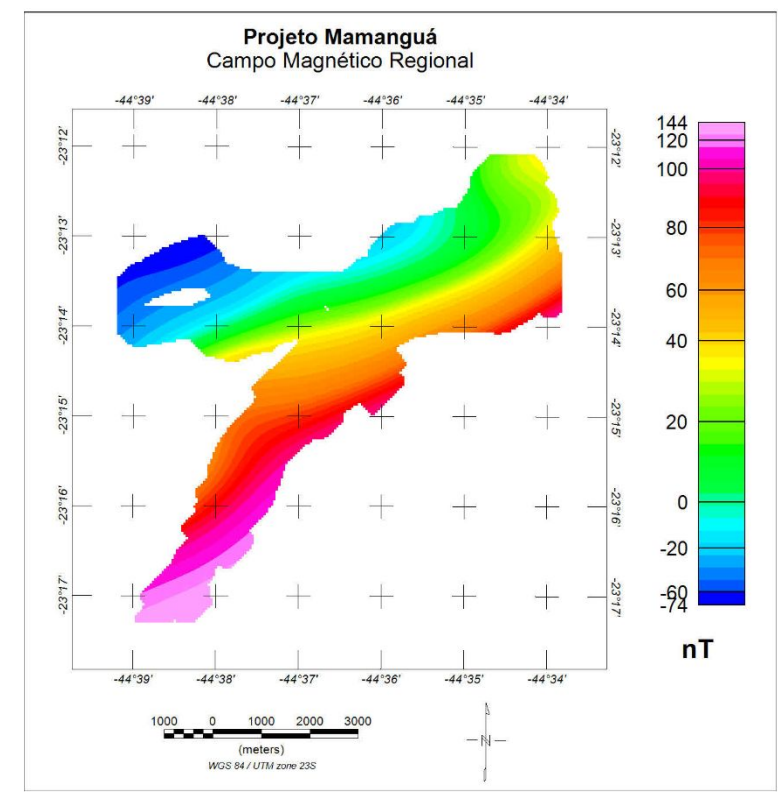

Figura 6: Resultado do campo magnético regional através da aplicação do filtro continuação para cima de $1500 \mathrm{~m}$ para os dados marinhos na área de estudo.

$\mathrm{Na}$ Fig. 7, o mapa do campo magnético residual apresentado foi obtido através da diferença entre o campo magnético corrigido do IGRF e o campo magnético regional. $O$ procedimento ressaltou as anomalias magnéticas de curto comprimento de onda localizadas na porção mais superficial da crosta terrestre. Os polígonos A e B destacam duas anomalias que apresentam tipos de polaridade diferentes. 
A anomalia do polígono A apresenta polaridade inversa, típica de uma magnetização remanente. O polígono $\mathrm{B}$ compreende uma anomalia com polaridade normal associada a uma magnetização induzida. Estas observações permitem inferir duas hipóteses: 1) as fontes de anomalias têm idades diferentes, sendo que uma delas foi gerada em época de magnetização reversa ; 2) as fontes anômalas têm a mesma idade, mas são compostas de materiais geológicos diferentes, sendo que a fonte no polígono B não contém materiais ferromagnéticos, como magnetita, maghemita, pirrotita e goetita.

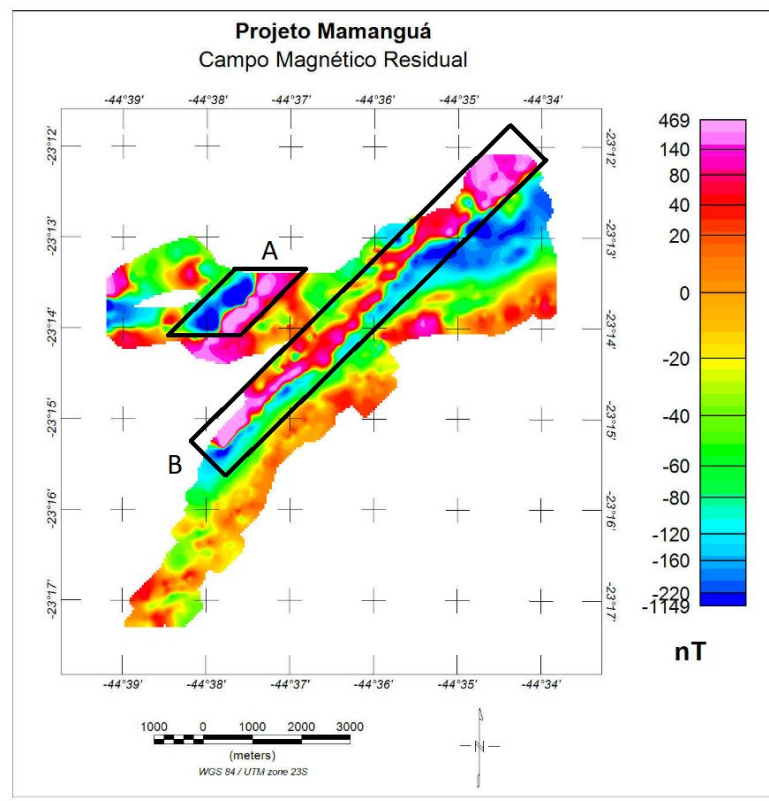

Figura 7: Representação do campo magnético residual com as áreas alvos. $O$ polígono $A$ ressalta uma anomalia magnética com polaridade inversa, enquanto o polígono $B$ se refere a uma anomalia com polaridade normal.

Na Fig. 8 apresentamos o mapa do campo magnético regional dos dados aeromagnetométricos obtido através da análise do espectro de potência, em que foi utilizado o filtro gaussiano regional com desvio padrão de $0,04 \mathrm{nT}$. O filtro gaussiano de separação regional/residual opera como um passa-banda, mas os dados de saída mostram uma distribuição gaussiana e o ponto de corte definido é o desvio padrão da função gaussiana (Oliveira, 2008). Com o intuito de preservar o maior conteúdo possível de altas frequências (fig. 9) as tendências regionais não foram extraídas ao máximo (fig. 8).

Como podemos observar na Fig. 9, o polígono da área de aquisição marinha abrange uma pequena região da aquisição aérea. As anomalias magnéticas notadas nos dados marinhos e aéreos apresentam padrões de lineamentos magnéticos orientados na direção NE/SW. Os dados aeromagnetométricos mostram que as anomalias observadas nos dados marinhos existem e são mais extensas do que mapeado dentro das enseadas.

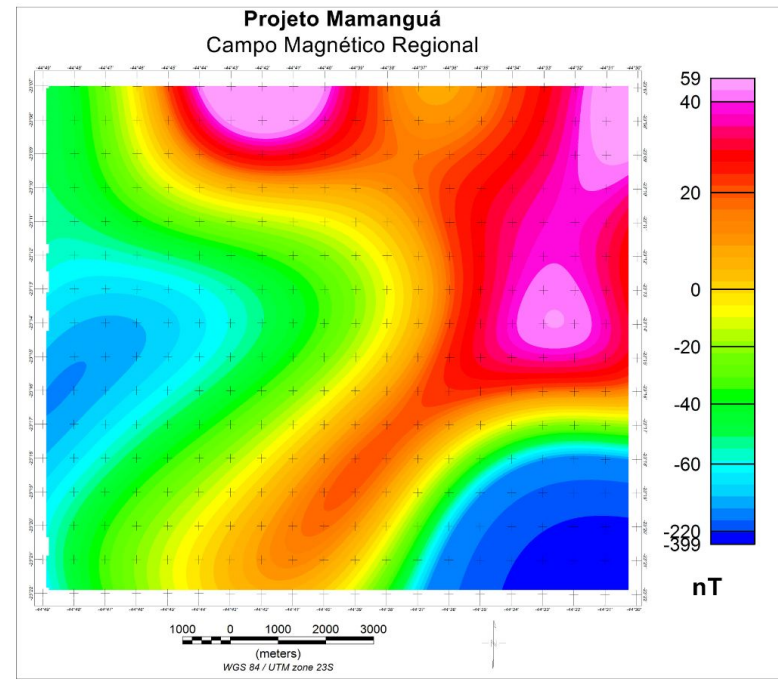

Figura 8: Resultado do campo magnético regional por meio da aplicação do espectro de potência com a utilização do filtro gaussiano regional.

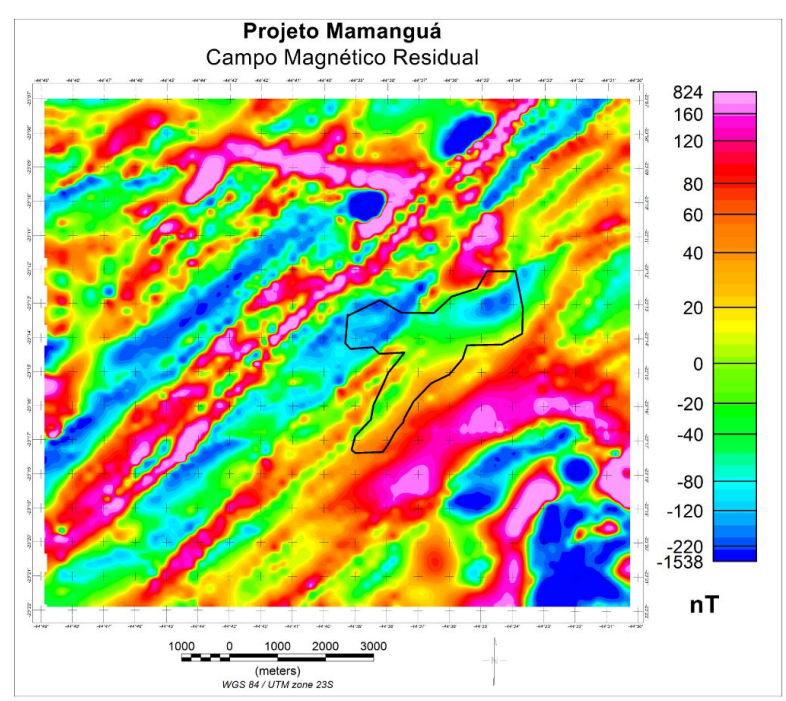

Figura 9: Representação do campo magnético residual. 0 polígono em linha contínua preta acima mostra a área de aquisição marinha.

Para os dados marinhos, utilizamos o programa de Inversão do Vetor de Magnetização com células de $X=150 \mathrm{~m}, \quad Y=150 \mathrm{~m}, \mathrm{Z}=100 \mathrm{~m}$ e considerando que $\mathrm{o}$ corpo-alvo variava em tamanho e profundidade. $O$ tamanho ideal da célula não é uma função do espaçamento de linha e, sim, uma função do espaçamento fonte-sensor (Schmick, 2018).

Como a área apresenta uma resposta mais uniforme, o erro absoluto de $8 \%$ fornece um desajuste com uma distribuição mais regular. Este tipo de erro tende a ajustar melhor as anomalias maiores (Schmick, 2018).

Com o propósito de refinar o modelo, modificamos alguns vínculos como a Inversão de Reponderação Iterativa (IRI) Focus e o ajuste de pesos do gradiente nas direções laterais (N-S e E-W) e na direção vertical. $O$ fator do IRI Focus foi alterado de 2 para 3. As alterações destacaram a distribuição de suscetibilidade positiva e negativa do modelo para indicar a possível 
presença de magnetização reversa na área de interesse.

Como o mapa de anomalia magnética indica várias feições lineares na direção NE/SW na área de estudo, para as direções laterais escolhemos um valor de 0,7 , razoavelmente próximo de 1 , indicando uma transição de célula para célula mais suave. Enquanto que para direção vertical, optamos por 0,5 para mostrar essa variação dos lineamentos.

Na Fig. 10 apresentamos o modelo obtido. Observe que as anomalias separadas nos polígonos resultaram em suscetibilidade expressiva e corpos alojados em subsuperfície. Talvez o corpo a NE esteja aflorando ou recoberto por pouca espessura sedimentar. Note que a direção média dos vetores de magnetização são diferentes para as duas áreas, reforçando a magnetização reversa e induzida.
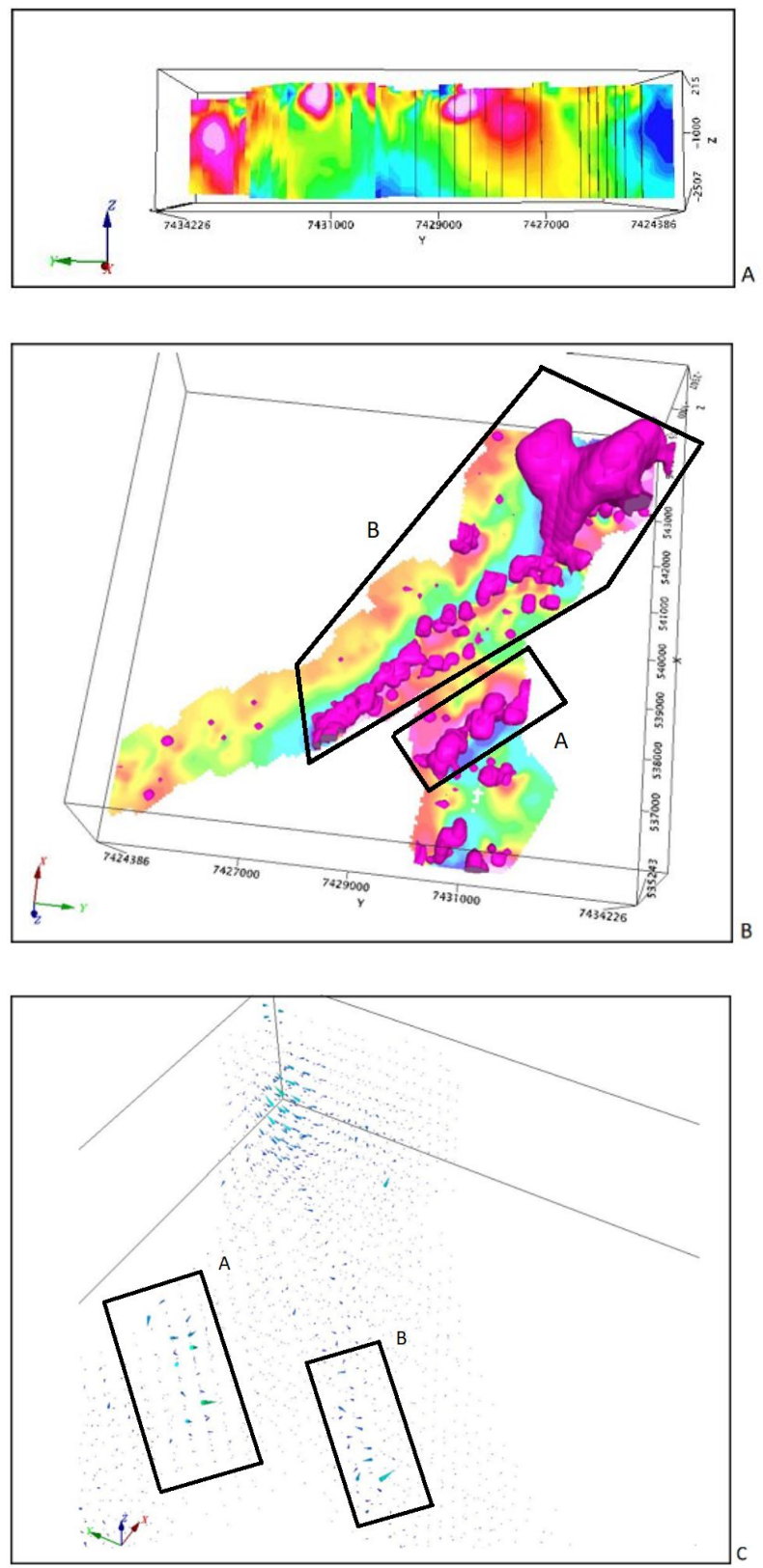

Figura 10: Resultado da Inversão do Vetor de Magnetização para a área de estudo: (A) MVI, (B) Isosuperfície de 0,009 SI e (C) direção e intensidade dos Vetores de Magnetização.
Para os dados aeromagnetométricos, as células utilizadas na Inversão do Vetor de Magnetização foram de $X=300 \mathrm{~m}, Y=300 \mathrm{~m}, Z=200 \mathrm{~m}$. Como a área de aquisição $\left(47.321 \mathrm{~km}^{2}\right)$ destes dados possui uma grande faixa de variação da anomalia, foi útil trabalhar com o erro relativo; pois, assim, características mais sutis foram realçadas.

O erro relativo tende a atribuir um menor erro às anomalias menores, ou seja, a inversão é forçada a atribuir erros maiores às anomalias maiores (Schmick, 2018). Quanto maior o erro, maior é a diferença entre os dados observados e os dados previstos pelo modelo. Optamos por um erro relativo de uma fração de $3 \%$ de cada medida observada, sendo que a fração padrão é de $5 \%$ para os sistemas em geral.

O único vínculo modificado foi o IRI Focus, que foi alterado para 3, evidenciando as propriedades físicas positivas e negativas do modelo.

As definições globais de aceleração e regularização foram alteradas para acelerar o processo de inversão e definir o método de ajuste, respectivamente. Limitamos o máximo domínio de influência dos elementos do modelo, acelerando os cálculos e mantendo um impacto insignificante sobre o resultado geral.

Os resultados da inversão dos dados aeromagnéticos encontram-se na Fig. 11. Podemos ressaltar que as anomalias estão bem representadas por fontes anômalas, mas as variações na direção de magnetização parecem menos significativas (fig. 11C), possivelmente, devido ao tamanho da célula utilizada na inversão.

As inversões dos dados marinhos e aéreos mostraram a existência de remanência (fig.10C), (fig. 11C). O polígono $A$, dipolo invertido, indicou forte presença de magnetização remanescente. Enquanto o polígono $B$ caracterizado por um dipolo normal indicou baixa presença de magnetização remanescente. Há um significativo contraste de susceptibilidade magnética aparente entre as anomalias de interesse (polígono $\mathrm{A}$ e B) e as anomalias que as circundam.

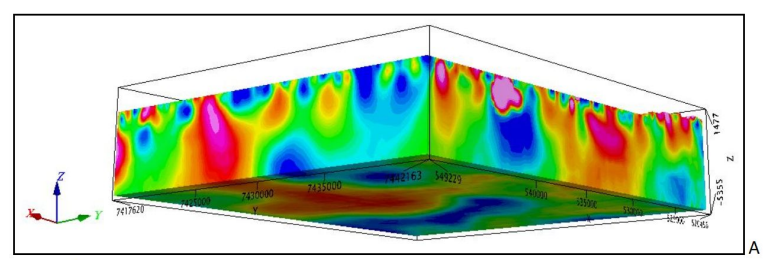



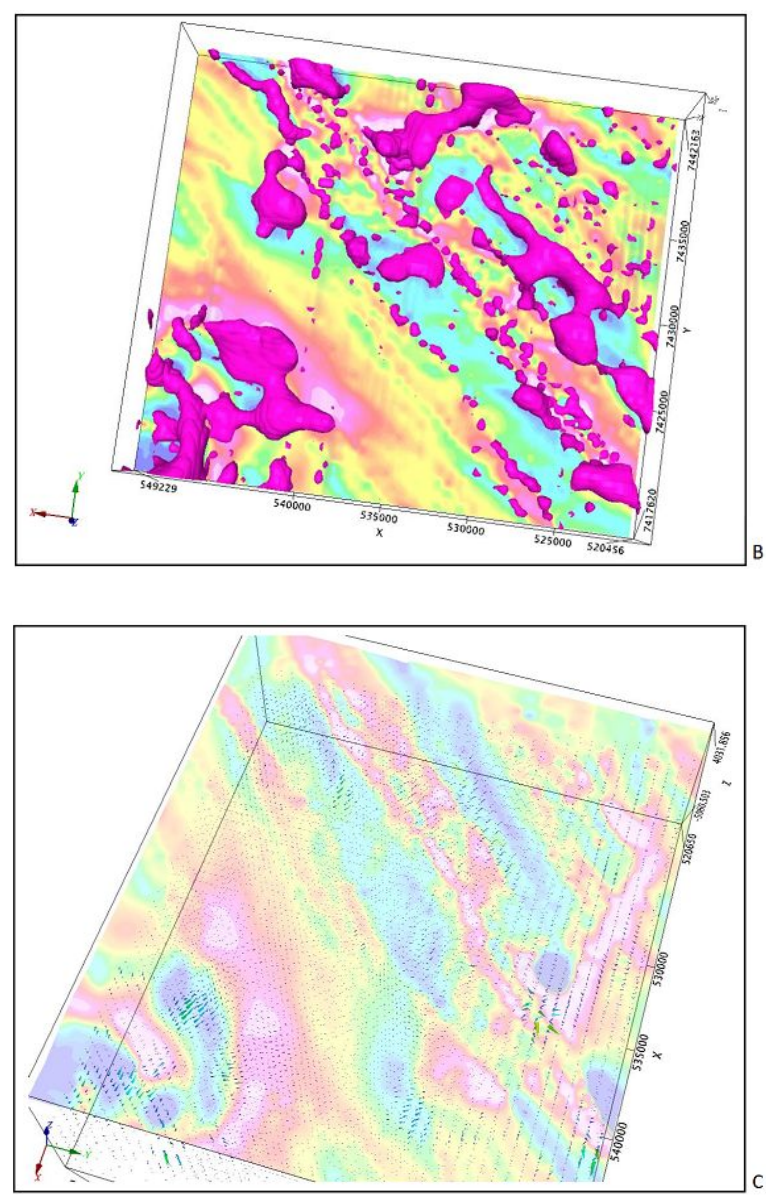

Figura 11: Resultado da Inversão do Vetor de Magnetização para os dados aeromagnetométricos: (A) MVI, (B) Isosuperfície de $0,02 \mathrm{SI}$ e $(\mathrm{C})$ direção e intensidade dos Vetores de Magnetização.

\section{Conclusions}

Destacamos duas zonas com características magnéticas distintas, advindas de corpos magnetizados em épocas diferentes ou com composição mineralógica bastante diferente. Notamos anomalias magnéticas com padrões de lineamentos na direção NE/SW que se estendem além da área de interesse, como observado nos dados de aerolevantamento. A interpretação qualitativa dessas feições lineares através do MVI indicou a presença de uma forte remanência no polígono $A$.

\section{Agradecimentos}

Agradecimentos à Geosoft Latinoamérica, pelos recursos fornecidos, ao Roberto Zanon, pela colaboração técnica, à Denise Moura, pelas discussões e por compartilhar seu conhecimento da área, e às instituições CPRM e CODEMIG pela disponibilização de dados geocientíficos.

\section{References}

ALMEIDA, F. F. M., CARNEIRO, C. D., 1998. Origem e evolução da Serra do Mar. Revista Brasileira de Geociências. 28(2):135-150.

BARBOSA, R.D \& PEREIRA, J.G. Inversão 3D de dados Magnéticos na Região de Mara Rosa - Goiás, Brasil, utilizando Geosoft VOXI. Thirteenth International Congress of the Brazilian Geophysical Society, Rio de Janeiro. 2013.

BERNARDES, R. A. Estrutura e dinâmica da ictiofauna do Saco de Mamanguá (Paraty-RJ). Tese de doutorado. Instituto Oceanográfico. Universidade de São Paulo. São Paulo, 1995.

BLAKELY, R. J., 1995. Potential theory in gravity and magnetic applications. Cambridge University Press, Cambridge, $441 \mathrm{p}$.

CPRM/CODEMIG. Relatório final do levantamento e processamento dos dados magnetométricos e gamaespectrométricos, área de $47.321 \mathrm{~km}^{2}$, região sudeste, Minas Gerais, Rio de Janeiro e São Paulo. 2013.

MAHIQUES, M. M. Considerações sobre os sedimentos de superfície do fundo da Baía de llha Grande, Estado do Rio de Janeiro. Dissertação de Mestrado. Volume I. Oceanografia Física. Instituto Oceanográfico. Universidade de São Paulo, 1987.

MALY. Avaliação da técnica da eletrorresistividade no mapeamento de sedimentos rasos associados a ocorrência de gás no Saco de Mamanguá e na Enseada de Paraty-Mirim. Dissertação de Mestrado. Instituto Oceanográfico. Universidade de São Paulo, 2017.

OLIVEIRA R.G. Arcabouço geofísico, isostasia e causas do magmatismo Cenozóico da Província Borborema e de sua margem continental (Nordeste do Brasil). Tese de doutorado. Programa de Pós-Graduação em Geodinâmica e Geofísica. Universidade Federal do Rio Grande do Norte, 2008.

OLPE, C. A. Hidrodinâmica e transporte de sedimentos no Saco de Mamanguá (RJ), observações e modelagem numérica. Dissertação de Mestrado. Programa de Pós-Graduação em Oceanografia, área de Oceanografia Geológica. Instituto Oceanográfico. Universidade de São Paulo, 2016.

SCHMICK. Top 5 Inversion Best Practices: Episode 1 Auxiliary Parameters. Geosoft Videos. 2018.

SMITH. Top 5 Inversion Best Practices: Episode 4 Interpreting MVI Components. Geosoft Videos. 2018.

TELFORD, W. M.; GELDART, L.P.; SHERRIFF, R.E.; KEYS, D.A. Applied Geophysics, Cambridge University Press. 1990. 